\title{
Médiévales
}

Langues, Textes, Histoire

56 | printemps 2009

Pratiques de l'écrit

\section{Les usages de l'écrit du Moyen Âge aux Temps modernes}

Entretien avec Roger Chartier. Propos recueillis par Étienne Anheim et Pierre Chastang

Roger Chartier, Étienne Anheim et Pierre Chastang

\section{CpenEdition}

Journals

Édition électronique

URL : https://journals.openedition.org/medievales/5564

DOI : $10.4000 /$ medievales.5564

ISSN : 1777-5892

Éditeur

Presses universitaires de Vincennes

Édition imprimée

Date de publication : 30 juin 2009

Pagination : $93-114$

ISBN : 978-2-84292-232-0

ISSN : 0751-2708

Référence électronique

Roger Chartier, Étienne Anheim et Pierre Chastang, « Les usages de l'écrit du Moyen Âge aux Temps modernes », Médiévales [En ligne], 56 | printemps 2009, mis en ligne le 21 septembre 2011, consulté le 23 avril 2022. URL : http://journals.openedition.org/medievales/5564 ; DOI : https://doi.org/10.4000/ medievales.5564

Ce document a été généré automatiquement le 23 avril 2022.

Tous droits réservés 


\title{
Les usages de l'écrit du Moyen Âge aux Temps modernes
}

\author{
Entretien avec Roger Chartier. Propos recueillis par Étienne Anheim et \\ Pierre Chastang
}

Roger Chartier, Étienne Anheim et Pierre Chastang

\section{NOTE DE L'ÉDITEUR}

Transcription de Marc Lagorsse, avec le soutien du laboratoire ESR de l'Université Versailles/Saint-Quentin-en-Yvelines.

1 Au début de la leçon inaugurale de votre chaire du Collège de France "Écrit et cultures dans l'Europe moderne $»^{1}$, vous précisez que votre enseignement se situera "entre la fin du Moyen Âge et notre présent ». Quelle importance attachez-vous à l'inclusion des derniers siècles du Moyen Âge dans la définition de votre travail?

2 L'importance des derniers siècles du Moyen Âge réside tout d'abord dans la discussion autour de l'invention, au milieu $\mathrm{du} \mathrm{xv}^{\mathrm{e}}$ siècle, de la reproduction des textes par caractères mobiles et de la presse à imprimer. Bien qu'il existe des continuités morphologiques, typographiques entre le livre manuscrit et le livre imprimé, ce moment-là a pu être interprété dans une perspective de printing revolution, d'une césure radicale.

3 Le XIV ${ }^{\mathrm{e}}$ siècle, si l'on suit les idées fortes d'Armando Petrucci, constitue le moment où, pour des auteurs qui écrivent en vulgaire ou en latin - dans les genres canoniques du droit ou de la théologie -, se crée un concept nouveau, celui du libro unitario, objet qui renferme dans sa reliure des textes ou un texte qui ont un seul auteur. Armando Petrucci, ou certains de ses élèves, ont étudié cette première apparition pour Pétrarque $^{2}$, mais on retrouverait, pour des auteurs français comme Christine de Pisan ou René d'Anjou, une évolution identique qui crée une césure par rapport à la tradition du livre en miscellanées dominante à partir des $\mathrm{VII}^{\mathrm{e}}-\mathrm{VIII}{ }^{\mathrm{e}}$ siècles. 
4 L'émergence du nom propre procède de ce nouveau concept de livre - l'œuvrelivre - qui joue sur l'identité entre un objet et une œuvre - œuvre isolée ou corpus de textes qui font œuvre. C'est un point très important par rapport à tous les raisonnements qui ont posé, en termes de volonté auctoriale ou de catégorie juridique, la question de l'émergence de l'œuvre comme livre et du livre comme œuvre. L'identité entre texte et objet est liée au geste matériel et intellectuel de la reliure, qui peut dépendre du possesseur du livre comme du copiste.

5 Toujours selon Armando Petrucci, les $\mathrm{XII}^{\mathrm{e}}$-XIII ${ }^{\mathrm{e}}$ siècles, au moins dans l'Europe méditerranéenne, représenteraient une rupture inauguratrice: celle du scrivere per leggere. À un modèle monastique de la copie, celle du scrivere senza leggere, s'oppose un modèle scolastique de la lecture où, à l'intérieur du monde universitaire, comme dans les mondes laïques séculiers apparaît le groupe des alfabeti liberi qui écrivent et lisent en dehors d'une obligation professionnelle.

6 Il y a donc, pour un moderniste, trois grandes raisons d'inclure la période médiévale, si l'on considère la périodisation classique du Moyen Âge s'achevant en 1492. La première est la révolution de l'imprimerie dans la décennie 1450 ; la deuxième est la naissance, au milieu du XIV siècle, du livre unitaire pour les textes en langue vulgaire et d'un concept moderne du livre qui lie étroitement le livre comme œuvre et l'objet comme livre; on aurait enfin ce moment de basculement, dans les sociétés occidentales, des rapports entre écriture et lecture, l'émergence d'une familiarité de lecture avec ce qui est copié, le déplacement des lieux de copie des textes, du scriptorium à la boutique du stationnaire, et un changement dans les méthodes de lecture. Dans un article, Franco Alessio analyse ce tournant fondamental des $\mathrm{XII}^{\mathrm{e}}$-XIII ${ }^{\mathrm{e}}$ siècles tant du point de vue des méthodes d'enseignement de la lecture, des lieux de production, que de la morphologie du livre, avec l'apparition du libro da banco, le grand livre de la scolastique ${ }^{3}$.

7 Toutes raisons qui doivent conduire à intégrer des lectures sur le Moyen Âge dans une réflexion de plus longue durée.

8 Tout au long de vos travaux, vous faites de fréquentes références aux historiens du Moyen Âge, en particulier à Armando Petrucci, auquel vous rendez hommage dans votre leçon inaugurale: pourriez-vous nous expliquer quelle importance a eue son œuvre, et plus généralement la bibliographie médiévale, dans le parcours d'un spécialiste de l'époque moderne comme vous?

9 Je l'ai beaucoup cité dès la première réponse. Je crois qu'il y a trois aspects importants dans l'œuvre d'Armando Petrucci, de ses élèves, et dans les publications de sa défunte revue Scrittura e civiltà. C'est tout d'abord de mettre en garde les modernistes contre une vision implantée par L'apparition du livre de Lucien Febvre et d'Henri-Jean Martin, paru en $1958^{4}$, qui assimile l'imprimerie, l'invention de Gutenberg, à la naissance du livre. Cette thèse a été renforcée par l'ouvrage d'Elizabeth Eisenstein, The Printing Press as an Agent of Change $e^{5}$, plus encore par la version courte ${ }^{6}$ qu'elle a donnée de ce premier ouvrage. Contre cette idée d'une rupture radicale, l'accent doit être mis sur les continuités morphologiques de la hiérarchie des formats qui correspondent à des types de discours (libro da banco, livre humaniste, libretto da mano, libro da bisaccia), et dont hérite directement le monde de l'imprimerie. Il s'agit également d'une continuité graphique, puisque aussi bien la lettre romaine que l'italique trouvent leur origine dans le monde du manuscrit et non dans les ateliers typographiques. Ajoutons une dernière forme de continuité, celle du livre unitaire que l'imprimerie se contente de renforcer, sans que disparaissent pour autant les miscellanées qui survivent et fleurissent même à 
l'âge de l'imprimé et auxquels l'historien moderniste doit par conséquent porter attention. Voilà le premier grand enseignement de la lecture par un historien moderniste des grands essais d'Armando Petrucci, particulièrement ceux publiés dans les volumes de la Letteratura italiana dirigée par Alberto Asor Rosa.

10 La deuxième raison c'est le fait que, peut-être de manière plus discrète mais dans des articles très aigus, Armando Petrucci a attiré l'attention sur les parentés morphologiques entre les écritures documentaires et les formes de l'écriture poétique ou de fiction. Il a ainsi consacré un article au rapport entre les formules de l'écriture notariée et les manuscrits autographes d'auteurs dont on dispose pour les $\mathrm{XIV}^{\mathrm{e}}-\mathrm{XV}^{\mathrm{e}}$ siècles ${ }^{7}$. D'une manière comparable à l'architecture gothique et la pensée scolastique chez Erwin Panofsky, ils sont liés par un substrat social commun: le monde des notaires. Beaucoup de ces auteurs sont fils de notaire ou notaires eux-mêmes, ce qui incite à envisager la culture écrite dans un continuum qui permet de saisir, dans une même visée, des écritures à fin esthétique, que l'on va appeler littéraires, et des écritures dites ordinaires, pratiques ou documentaires. Le troisième élément, c'est qu'Armando Petrucci a inventé des thèmes de recherche qui se sont imposés comme importants dans le monde des modernistes, comme le scrivere per gli altri ou les délégations d'écriture, qui invitent à comprendre qui écrit pour qui dans une relation hiérarchique, professionnelle ou dans une relation d'homogénéité sociale. Ajoutons les compétitions sur la norme graphique entre les calligraphes professionnels, les secrétaires et les maîtres d'écriture, étudiées dans son livre La Scrittura ${ }^{8}$. La question également de l'expertise sur les écritures, particulièrement quand il s'agit de reconnaître les mains des écritures infamantes et diffamatoires. Pour tous ces thèmes, des travaux ont été menés par Armando Petrucci ou certains de ses élèves, comme Laura Antonucci. On peut ainsi dire qu'Armando Petrucci a d'emblée fait ce franchissement prospectif, puisqu'il a intégré dans sa compétence de paléographe des écritures médiévales, de multiples réflexions et travaux sur le premier âge moderne. On peut également penser au livre que je viens de citer sur La Scrittura, titre un peu excessif puisqu'il traite des écritures exposées dans la très longue durée, depuis le moment de leur retour aux $\mathrm{XI}^{\mathrm{e}}$-XII ${ }^{\mathrm{e}}$ siècles jusqu'aux graffitis de mai 68. En même temps, Armando Petrucci est un nom qui peut dépasser la personne dans la mesure où, pour moi, il incarne l'ensemble de travaux essentiellement italiens qu'il a inspirés, et qui ont été publiés en particulier dans la revue Scrittura e civiltà.

11 Je pense ainsi que l'intérêt à l'heure actuelle porté à une certaine forme d'histoire de l'écriture dans le monde espagnol est un héritage des travaux ou de l'enseignement d'Armando Petrucci pour un certain nombre de collègues, d'un côté Francisco Gimeno Blay à Valence et de l'autre Antonio Castillo Gómez à Alcalá de Henares; le premier est un spécialiste des écritures médiévales castillanes ou catalanes, mais avec les mêmes perspectives que celles d'Armando Petrucci; le second est quant à lui spécialiste des écritures des $\mathrm{XVI}^{\mathrm{e}}$ et $\mathrm{XVII}{ }^{\mathrm{e}}$ siècles.

Ma rencontre avec l'inspiration d'Armando Petrucci a été d'emblée très heureuse car il n'a pas voulu limiter l'histoire de l'écriture au domaine de la paléographie médiévale, dont il était un maître, mais il a cherché à entrer toujours plus en avant dans l'époque moderne jusqu'au contemporain; peut-être parce qu'il y avait chez lui un intérêt puissant pour les problèmes du présent, dans une perspective que l'on peut qualifier de progrès, et un engagement civique et politique, rare en France et qui, généralement, ne va pas de pair avec l'exercice paléographique ! D'où ses livres portant sur l'écriture, les 
pratiques de l'écrit et les inégalités dans l'accès à l'écrit dans le monde contemporain, et cette volonté de regarder en amont, à partir du thème de l'apparition du livre ou de la printing revolution.

13 Le développement de la réflexion des médiévistes dans le domaine de la culture de l'écrit s'est largement nourri des travaux de l'anthropologie britannique de la fin des années 1960 - je pense en particulier à Jack Goody ${ }^{9}$-, dont la démarche a profondément influencé un historien comme Michael Clanchy ${ }^{10}$. Quel rôle ces travaux ont-ils joué dans votre propre parcours?

La perspective de Jack Goody est double. Il s'agissait premièrement d'une perspective critique dans l'anthropologie contemporaine, de montrer que les opérations auxquelles les anthropologues, en particulier structuralistes, soumettaient les mythes ou les rites, produisaient une très importante distorsion. La seconde perspective consistait à mettre en évidence les mutations historiques dans les manières de penser occasionnées par l'apparition de la fixation écrite. Il s'agit d'un acquis très largement partagé.

15 Sur deux éléments plus particuliers, je vais essayer de répondre à la question du lien entre Jack Goody et Michael Clanchy, puisqu'effectivement ce dernier décrit un monde de l'oralité qui, avec l'intervention de l'inscription écrite à la fin des pratiques judiciaires ou son usage dans l'administration, se déplace vers un monde des written records. Du coup, ce qui est en question, c'est le rapport avec la parole vive, c'est-à-dire l'authenticité plus ou moins forte donnée au témoignage oral par rapport à l'enregistrement écrit et, plus généralement, l'importance donnée à la procédure écrite dans le monde de la justice par rapport à la force de la parole. Puisqu'il y a des témoins, se pose la question de la transcription de la parole, de ce qui a été dit, et des médiations à l'œuvre dans ce processus. Mais se trouve aussi posé un thème plus fondamental pour la première modernité : celui de la nostalgie du primat perdu de la parole vive, dans un monde de la prolifération de l'écrit.

16 J'ai utilisé cette perspective d'une manière très précise dans un essai qui porte sur la seconde partie du Henry VI de Shakespeare ${ }^{11}$, dans laquelle est mise en théatre une rébellion effective, celle de Jack Cade en 1450. Ce fait historique avéré est réinterprété par Shakespeare d'une double manière. De cette rébellion de 1450, qui utilisait massivement l'écrit puisqu'elle est connue en particulier par les pétitions au roi des rebelles dénonçant les mauvais ministres et les méchants officiers, Shakespeare donne une interprétation millénariste et prophétique, à l'image de la rébellion de 1381. Mais il lui prête aussi un discours radical de négation de l'écrit, considéré comme une forme d'oppression. Du coup, dans le monde utopique ou uchronique de Cade, l'écrit est banni, l'imprimerie, avec un léger anachronisme, est dénoncée, et la parole vive devient l'unique forme du gouvernement et de la justice. Ce qui est très intéressant dans cette représentation, c'est évidemment son statut ambigu: que pouvait-elle signifier pour les spectateurs ou les lecteurs de la fin du XVI ${ }^{\mathrm{e}}$ et du début du XVII ${ }^{\mathrm{e}}$ siècle ? C'est une question complexe puisque Cade reprend en charge toute une tradition de valorisation de la parole vive, mais dans un personnage manipulé, grotesque et cruel. Il y a donc une ambivalence, une ambiguïté, ce texte, comme tous ces textes de théâtre, est ouvert à la pluralité contradictoire des interprétations. D'autre part, il est très curieux que Shakespeare fasse dire à Cade, dans l'anglais de la fin du Xvi siècle, une formule qu'on trouve dans le droit canonique : «peut-on se fier à la peau d'un animal mort? " qui devient, dans le cas de Cade, "peut-on se fier à la peau d'un innocent agneau? ", renforçant de la sorte la dimension christique du support. Voilà un exemple du rapport entretenu avec la parole vive au sein de l'inexorable processus qui fait 
rentrer la justice, l'administration et les relations, quelles qu'elles soient, dans le monde de l'écrit.

17 Un deuxième élément peut être ajouté. Pour Jack Goody, les premières formes d'écriture sont des mises en liste d'objets, d'êtres, de concepts. Il me semble que parfois, dans certaines situations à l'âge moderne, on peut être inspiré par cette problématique. Je pense par exemple à la rédaction des cahiers de doléances. J'avais écrit, il y a bien longtemps ${ }^{12}$, un article sur les cahiers de doléances de 1789 qui essayait d'utiliser cette problématique, tout en s'inspirant également de la scène de 1789 d'Ariane Mnouchkine qui montrait un chaos de revendications mis en ordre par un scribe ou un notaire. Ces derniers introduisaient une hiérarchie, des classements, un ordre des préférences. Inspiré par cette interprétation de théâtre et par les réflexions de Jack Goody, on peut essayer de penser le travail de mise en listes à l'époque moderne, à la jonction entre la spontanéité de l'expression orale et son nécessaire ordonnancement dans des documents qui acquièrent un statut officiel, ici la consultation politique de 1789 . On pourrait dire la même chose pour les cahiers de doléances de 1614 .

Ces deux micro-exemples montrent, je crois, que l'on peut parfois lire les auteurs pour deux raisons. D'abord pour l'ampleur de la thèse, et celle de Jack Goody est particulièrement forte. Elle porte, d'une part, sur ce que produit l'introduction de l'écrit et de l'écriture alphabétique dans une société, qu'il s'agisse de la Mésopotamie ou de certaines ethnies africaines. Elle apporte, d'autre part, une connaissance sur les schèmes de pensée propres à l'écrit qui, lorsqu'ils s'appliquent à des énonciations comme les mythes ou à des structures du comportement comme celles des rites, organisent des hiérarchies sous forme de listes ou des oppositions dualistes sous forme de tableaux, produisant du coup à la fois connaissance et déformation. Mais on peut aussi utiliser ces textes fondamentaux à l'intérieur d'une analyse plus particulière, et produire peut-être une interprétation qui n'avait pas été aussi fortement marquée auparavant.

19 À l'occasion, vos recherches ont même directement porté sur les pratiques de l'écrit au Moyen Âge, qu'il s'agisse de Baudri de Bourgueil ${ }^{13}$ dans Inscrire, effacer ${ }^{14}$, ou dans plusieurs chapitres de Culture écrite et société15. Quelle expérience tirez-vous de ces incursions?

La première expérience est la mesure de mon immense ignorance! Pourquoi Baudri de Bourgueil ? Pour deux raisons ; d'abord, la lecture de Ernst R. Curtius. Dans ce fameux chapitre consacré au livre et à ses usages métaphoriques au Moyen Âge et à la Renaissance - de Bonaventure à Shakespeare et Galilée ${ }^{16}$-, il écrit quelques pages sur cet abbé poète qui prend pour thème, dans un certain nombre de ses poèmes, les pratiques et les objets même de son écriture, proposant ainsi une espèce de mise en abyme de l'écriture dans l'écriture. Or, un des projets qui ont donné naissance à Inscrire et effacer était d'essayer de repérer comment certains textes, que l'on peut qualifier de littéraires, proposaient, pour produire des effets esthétiques, ironiques ou critiques, une mise en représentation de l'écriture et une mobilisation des objets et des pratiques utilisés pour leur propre publication. La lecture du livre de Jacqueline Cerquiglini, La couleur de la mélancolie ${ }^{17}$, avait déjà attiré mon attention sur les textes médiévaux par l'intérêt, proche du mien, porté à la mise en écriture de l'histoire de l'invention de l'écriture et d'une première canonisation d'un répertoire d'auteurs, qui accompagnait assez bien la double histoire du livre unitaire et de l'émergence de la fonction auteur. 
21 La deuxième raison qui a rendu possible cette analyse, c'est évidemment la traduction qu'a donnée Jean-Yves Tilliette de Baudri de Bourgueil dans une édition en deux volumes qui a eu un sort malheureux, puisqu'une partie des exemplaires du premier tome a brûlé dans l'incendie des Belles-Lettres. Mon latin ne m'aurait pas permis d'écrire sur Baudri de Bourgueil. Je pense qu'on peut avoir une compétence réduite lorsqu'on utilise de manière marginale un texte dans une autre langue, mais quand on en fait l'objet même de l'analyse, il faut avoir un rapport plus sérieux avec la langue du texte. Lorsque ce rapport n'est pas assuré, une édition bilingue s'avère nécessaire.

Baudri de Bourgueil renvoie à une question, qui a été peut-être un peu négligée par l'histoire de l'écriture y compris par les grands comme Armando Petrucci, à savoir celle des écritures éphémères, des écritures transitoires, des écritures qui ne sont pas destinées à demeurer. Évidemment pour une histoire de l'écrit, il vaut mieux avoir des sources et des traces analysables, mais cette histoire des objets qui portaient des écritures destinées à disparaître est fascinante, et Baudri de Bourgueil ouvrait largement cette perspective. Il décrivait en effet ses pratiques de composition sur la cire de tablettes, avant qu'un scribe ne les transcrive sur du parchemin. Dans un poème, il est totalement irrité par son scribe très paresseux qui ne copie pas assez vite ; ses tablettes sont pleines et il ne peut pas les effacer pour écrire de nouveaux vers.

Cela entrait en résonance avec un travail que j'avais commencé avec Peter Stallybrass pour comprendre ce qu'étaient, durant les $\mathrm{XVI}^{\mathrm{e}}$ et $\mathrm{XVII}^{\mathrm{e}}$ siècles, les writing tables ${ }^{18}$, dont on trouve de nombreuses attestations dans les textes eux-mêmes, dans les didascalies des œuvres du théâtre anglais, et que l'on a pu retrouver par la suite, par une recherche assez simple d'ailleurs. Comme certaines avaient été associées à des almanachs imprimés, elles avaient été classées dans le Short Title Catalogue des livres anglais des $\mathrm{XVI}^{\mathrm{e}}$ et $\mathrm{XVII}^{\mathrm{e}}$ siècles. Il s'agit d'objets sur lesquels les pages de papier étaient recouvertes d'une fine pellicule de colle, de vernis et de plâtre, qui permettait, comme sur la tablette de cire, d'écrire, puis d'effacer avec la salive ou un chiffon humide, afin de réécrire sur la même surface. Les writing tables avaient donc non seulement l'avantage d'économiser du papier, mais elles permettaient également d'écrire en dehors de la technologie assez complexe qui suppose des plumes, des couteaux pour les effiler, un encrier, du sable ou quoi que ce soit d'autre pour sécher l'écriture, une technologie qui rendait très difficile, aux $\mathrm{XVI}^{\mathrm{e}}$ et $\mathrm{XVII}{ }^{\mathrm{e}}$ siècles, l'écriture dans l'espace public. Les writing tables offraient cette possibilité. Cette recherche permettait d'éclairer un des monologues d'Hamlet, celui où le personnage, c'est-à-dire l'acteur qui joue le rôle, se réfère à " my tables, my tables ", lorsque le spectre lui demande d'écrire la seule chose qui importe : "remember me ». Dans ce monologue, la réflexion d'Hamlet commence avec la métaphore classique des tablettes de cire comme désignant la mémoire, une métaphore à l'antique. Mais, au Xvi siècle, les tablettes de cire n'étaient plus en usage. Du coup, les writing tables constituaient l'objet qui pouvait donner existence sur la scène à cette métaphore. Non seulement Hamlet déclare qu'il va effacer des tablettes de sa mémoire tout savoir inutile pour que seule subsiste l'injonction du spectre, mais il sort alors de sa poche un objet sur lequel il commence à écrire. Ce passage appelait à repérer les jalons d'une histoire des objets de l'écriture transitoire ou éphémère, en étant attentif à la discontinuité de ces supports. Les tablettes de cire, nombreuses à l'époque médiévale, n'ont plus que des usages marginaux aux $\mathrm{XVI}^{\mathrm{e}}$ et $\mathrm{XVII}{ }^{\mathrm{e}}$ siècles; en revanche se diffusent des objets faits de papier préparé qui permettaient d'effacer et de réécrire, et que l'on trouvait non seulement dans le monde anglais avec les writing 
tables, mais aussi dans le monde espagnol avec les librillos de memoria. Puis aux $\mathrm{XIX}^{\mathrm{e}}$ et $\mathrm{xx}^{\mathrm{e}}$ siècles, apparaît ce que l'on appelle en allemand Wunderblock, un objet sur lequel est placée une pellicule de cellophane qui permet d'écrire et d'effacer par un système mécanique et auquel Freud a consacré un essai fameux ${ }^{19}$. C'est un objet que j'ai moimême utilisé! Il y avait là une ébauche d'histoire des écritures transitoires et éphémères inspirée en grande partie par ce travail sur Baudri de Bourgueil ; histoire qui constitue évidemment un défi pour tout historien puisque les documents sont très rares. Pourquoi des writing tables ont-elles été conservées? Parce qu'à la fin du $\mathrm{XVI}^{\mathrm{e}}$ siècle des relieurs à Londres ont eu l'idée qu'ils pouvaient enchâsser les feuillets destinés aux écritures transitoires et éphémères dans des pages sur lesquelles étaient imprimés un calendrier, les dates des foires, les distances entre les villes, le cours des monnaies... Considérées d'emblée comme un objet imprimé, elles ont été conservées ; on les trouve à la British Library ainsi que dans beaucoup de bibliothèques universitaires anglaises et américaines.

Au-delà de cette expérience directe, je plaide pour l'idée qu'on ne peut pas s'enfermer dans des temps, dans des lieux, dans des langues; en même temps, parce que certaines tentations ou réalisations sont un peu risquées, il ne faut travailler que sur ce que l'on peut contrôler, soit par une bonne connaissance de la bibliographie, soit en définissant, lorsqu'on est hors de son propre territoire, des objets qui sont contrôlables du point de vue scientifique.

Ces questions posent en général le problème de la périodisation; vous parlez vousmême de "repérer les durées sédimentées de la culture écrite pour comprendre les mutations qui l'affectent dans le présent »: où placeriez-vous de telles inflexions, et pensez-vous que l'on puisse opposer Moyen Âge et Temps modernes à cet égard?

Non, je pense que l'on doit les englober dans la mesure où pour moi il y a trois ruptures essentielles qui accompagnent l'avènement de la culture imprimée : une rupture sur la morphologie des objets, celle qui porte sur la technique de reproduction des textes, et celle qui concerne les pratiques, les usages ou les appropriations des textes portés par des objets. Si l'on réfléchit en termes de morphologie la grande rupture - et cela mine dans un sens fondamentalement la thèse d'Elizabeth Eisenstein -, c'est l'apparition du codex entre les $\mathrm{II}^{\mathrm{e}}$ et $\mathrm{IV}^{\mathrm{e}}$ siècles qui modifie radicalement la forme d'inscription des textes. Dans le rapport entre objet et texte, on assiste à une inversion que Guglielmo Cavallo a très bien montrée. Dans le monde des rouleaux, une même œuvre, si elle a une certaine ampleur, est inscrite sur plusieurs livres. J'utilise ce terme bien qu'en anglais, les gens répugnent à dire que les volumina sont des livres. Le terme de book est presque équivalent à celui de codex en français. La tolérance est plus grande en français où le terme de livre désigne, d'une manière générale, la forme d'un texte écrit qui a une certaine unité et cohérence. Il existe donc une pluralité de rouleaux pour le même texte. À l'inverse, progressivement, le codex devient le lieu de réception d'une pluralité de textes avec la forme dominante, complexe et variable selon les temps, des miscellanées. Les premiers siècles de l'ère chrétienne constituent donc une rupture absolument fondamentale qui pose par ailleurs le problème des survivances de la forme rouleau à l'âge du codex.

Une deuxième rupture intervient dans la technique de reproduction du texte. L'invention de Gutenberg - que j'ai tendance à limiter, à discuter mais ce serait une absurdité totale de penser que rien ne change avec la reproduction mécanique des textes - créait pour la première fois une nouvelle réalité. Dans le monde du rouleau ou 
dans celui du codex manuscrit, chaque objet est porteur d'une œuvre qui presque par nécessité introduit de la variance, alors que la reproduction mécanique, même si l'on doit être très prudent quant aux variations qui peuvent exister entre les exemplaires d'une même édition imprimée, dissémine dans une pluralité d'objets ce qui est considéré, non pas peut-être littéralement mais au moins intellectuellement ou esthétiquement, comme la même œuvre. Il y a donc une rupture tout à fait fondamentale introduite dans le registre de la reproduction.

Le troisième élément serait de considérer, avec la prudence nécessaire, les révolutions de la lecture, c'est-à-dire les transformations profondes qu'introduit une nouvelle morphologie du livre dans les relations avec le texte. Guglielmo Cavallo insiste ainsi sur les traits caractéristiques du rouleau, qui ne permet pas d'écrire en lisant, puisque les mains sont mobilisées par les supports sur lesquels il s'enroule. Pour composer en lisant, il faut passer par la dictée, l'oralité. Le codex quant à lui invite évidemment à écrire en lisant, hors ou dans le livre lu. Il est également impossible de feuilleter un rouleau; du coup tous les dispositifs de repérage du texte - la construction des instruments de type index, tables ou concordances - et la discontinuité de la lecture sont intrinsèquement liés au codex. Donc on a, d'un côté, des mutations des pratiques du texte qui sont dépendantes de la morphologie de l'objet, et l'on a, d'autre part, des transformations des pratiques du texte qui résultent de mutations culturelles. S'il y a une révolution de la lecture silencieuse, elle est liée à un processus de très longue durée qui enjamberait le Moyen Âge pour aller jusqu'au XIX ${ }^{e}$ siècle. Cette capacité pour des lecteurs de lire seulement avec les yeux et en silence est encore considérée comme pouvant discriminer illettrés et lettrés au XIX ${ }^{e}$ siècle. Si l'on suit avec prudence la thèse de Paul Saenger, cette capacité était exceptionnelle dans les premiers siècles du Moyen Âge, - même si la discussion menée en particulier par Guglielmo Cavallo et Armando Petrucci a montré qu'existait une pluralité des compétences de lecture dans le monde antique. Une autre transformation pourrait être celle de la révolution de la lecture au XVIII ${ }^{\mathrm{e}}$ siècle, concept refusé par Robert Darnton, mais accepté par beaucoup d'historiens allemands, et qui constituerait également une transformation culturelle en termes de pratiques, d'autorité du texte, d'un rapport plus critique ou plus désinvolte instauré avec le texte, en termes également d'abondance et de prolifération de l'écrit.

On a des séries chronologiques qui, sans être synchrones, fonctionnent sur des registres parallèles, puisque les transformations et les mutations morphologiques, techniques et culturelles ont leur durée propre, dans laquelle se dissolvent les frontières non seulement entre Moyen Âge et époque moderne, mais également entre première modernité et monde contemporain.

Donc, je pense que l'on doit réfléchir sur « les durées sédimentées de la culture écrite », qui constituent également un élément pour comprendre le présent, parce que pour la première fois, avec la révolution numérique, les trois transformations seraient synchrones. Le codex a été inventé dans un monde où les textes ont continué à être reproduits à la main ; l'imprimerie a constitué une nouvelle technique de reproduction des textes dans un monde où la morphologie du livre n'a pas changé ; les révolutions de la lecture se situent quant à elles dans des contextes de stabilité morphologique ou technique. La révolution de la lecture silencieuse intervient dans le monde du codex et la révolution de la lecture extensive du xvIII ${ }^{e}$ siècle - pour reprendre une formule peut-être un peu discutable - ou la révolution de la démocratisation de la lecture au $\mathrm{XIX}^{\mathrm{e}}$ siècle se produisent dans le monde de l'imprimé. 
31 Or, le monde digital est un monde qui se caractérise par une nouvelle technique de reproduction, de dissémination et de réception des textes. À cela s'ajoute une nouvelle morphologie pour l'inscription des textes sur un nouveau support, jusqu'à maintenant l'écran. Et il y aurait sans doute à observer plus finement des transformations profondes dans les pratiques de la lecture. Si, comme l'a montré Peter Stallybrass, la morphologie du codex permet cette lecture discontinue que le rouleau rendait difficile - passer d'une page à un autre, comparer un fragment du texte avec un autre, utiliser les index pour des lectures fragmentées -, la lecture discontinue, fragmentée et segmentée face au texte électronique diffère de celle face au codex manuscrit ou imprimé, en cela que la totalité du livre, entendu au sens d'œuvres ou de textes rassemblés au sein d'une même unité, est imposée par la matérialité de l'objet. Une relation même inconsciente s'opère immédiatement entre le fragment et la totalité dont il est extrait par la lecture. Or cette opération est rendue purement abstraite dans le monde du texte électronique, puisque la totalité n'est perceptible que par des mesures de bytes; elle n'est pas donnée dans la matérialité de l'objet et confère de ce fait un autre sens à la lecture fragmentée, surtout lorsqu'elle n'est pas venue à l'écran à partir du codex imprimé. Les textes risquent alors de se transformer en une sorte de banque de données, dont on extrait des informations, sans avoir à les assigner ou à les relier avec la totalité de laquelle elles sont extraites. La discussion sur la périodisation mène donc au temps présent.

D'une manière plus générale, l'importance croissante accordée par les historiens à la dimension matérielle du texte et aux effets de sens induits par la forme a conduit à un accroissement progressif de l'usage des savoirs produits par les disciplines de l'érudition du texte. Comment envisagez-vous, dans la pratique historique comme dans le champ académique, la redéfinition des relations entre l'histoire et ces disciplines érudites?

Je pense qu'au sein de l'histoire intellectuelle et culturelle définie dans une conception moderne, ces disciplines, souvent traitées dans les manuels comme disciplines auxiliaires, deviennent absolument centrales. Elles deviennent centrales parce qu'elles permettent de refuser à la fois une idéalité sans historicité, et en même temps une approche structurale sans matérialité. Je m'explique. Ces disciplines commencent avec l'étude des objets. La paléographie et la codicologie comme la bibliographie matérielle commencent avec des descriptions formalisées et rigoureuses des objets, qui renvoient finalement aux procédures de leur production. Mais ces objets sont porteurs fondamentalement d'écrits. Les descriptions minutieuses et codifiées des œuvres imposent donc un cadre à l'interprétation du texte. On a vu avec la codicologie l'opposition entre miscellanées et libro unitario. La bibliographie matérielle a, quant à elle, élargi son questionnement ; au savoir descriptif centré sur les objets imprimés, qui s'attachait à reconstituer leur processus de publication, elle a progressivement ajouté la description des modes d'inscription du texte - division du texte sur la page, graphies des mots, ponctuation... Cet élargissement permet d'aller plus avant dans le rapport entre la matérialité de l'objet, décrite par la discipline d'érudition, et l'interprétation de la textualité qui résulte du travail de l'historien. Le savoir produit par les disciplines de l'érudition a une vertu fondamentale : il empêche la réduction des textes à un pur contenu sémantique, réduction que les approches structurales partagent avec la tradition plus classique de l'idéalité du texte. Toutes deux ont oublié que les formes matérielles contribuent, imposent, bornent la production de la 
signification et qu'il y a une très profonde historicité des textes matérialisée dans les variances, dont certains ont fait l'éloge pour la civilisation médiévale du manuscrit. Mais, dans le monde de l'imprimé, il existe aussi des variations avec la succession des éditions, comme entre les exemplaires d'une même édition. Il est donc nécessaire de réintroduire, dans l'interprétation, la mobilité, l'instabilité et l'historicité rendues visibles par une attention méticuleuse à la matérialité des textes. À partir de là, je pense que les disciplines auxiliaires deviennent absolument centrales parce qu'elles dissipent ces illusions. Par exemple, elles dissipent l'illusion que les lecteurs lisent les auteurs ; or les lecteurs lisent des objets écrits, qu'il s'agisse de copies manuscrites ou d'éditions multiples qui, dans les deux cas, supposent des médiations, des interventions, des techniques qui se multiplient avec l'imprimerie, mais qui sont également présentes dans le monde médiéval où un lecteur lit rarement un manuscrit autographe. La critique structuraliste a eu accès aux textes, en faisant abstraction de toutes les variations des textes eux-mêmes et de tous les agents auxquels ces variations peuvent être rapportées.

Si l'on suit le processus de publication d'un ouvrage aux $\mathrm{XVI}^{\mathrm{e}}$-XVII ${ }^{\mathrm{e}}$ siècles, les interventions sont multiples: un scribe copie au propre le manuscrit autographe, un censeur intervient dans le texte, un maitre imprimeur établit l'organisation du travail dans l'atelier, un libraire-éditeur décide du format, du tirage, de l'illustration, un correcteur établit le texte. Pèsent enfin des choix volontaires ou des contraintes de travail qui régissent les pratiques des compositeurs. On voit donc qu'on est à très grande distance de l'autographe, considéré traditionnellement comme ce qu'il faut retrouver même lorsqu'il a disparu. Cette obsession, en un sens paradoxal, de la bibliographie matérielle constitue également un fondement irréfléchi dans le travail de pans entiers de la critique littéraire. Or, comme disait un bibliographe ou un bibliothécaire américain, les auteurs n'écrivent pas les livres, pas même les leurs; ils écrivent des textes que de multiples opérations transforment en livres. La centralité de ces disciplines d'érudition qui décrivent ces objets et, à travers ces objets les procédures à l'œuvre et, à travers ces procédures la chaîne de tous ceux qui, en général anonymement, contribuent à cette relation entre le lecteur qui lit et l'auteur qui a écrit, me paraît essentielle.

C'est pour cela qu'à partir d'une compétence de bibliographie matérielle, Donald MacKenzie ${ }^{20}$ a ouvert à une sociologie des textes, en élargissant le champ des objets, puisqu'il propose de s'attacher aux non book texts, c'est-à-dire aux productions de l'imprimé situées au-delà des livres, poussant même l'argument jusqu'au non verbal texts, lorsque, métaphoriquement et peut-être avec un certain risque, le terme de texte est appliqué à des objets qui ne portent pas l'écriture mais qui font sens par la relation entre leurs éléments constitutifs. Cet élargissement du champ des objets se double d'un accroissement de ce que l'on doit prendre en compte dans la description bibliographique; la bibliographie matérielle était surtout préoccupée par l'identification hypothétique du manuscrit qui avait servi pour la composition typographique, par l'établissement d'une édition, si possible exemplaire, et par les descriptions rigoureuses qui permettent d'organiser bibliographies, catalogues et répertoires. Or il s'agit maintenant de prendre en compte ce qu'on appellerait aujourd'hui aux États-Unis the materiality of the text, c'est-à-dire les formes d'inscription du texte. On pourrait suivre cette histoire, depuis les deux colonnes du rouleau, les grands manuscrits scolastiques glosés et, comme l'a fait Anthony Grafton, l'apparition d'une nouvelle organisation ou structuration du texte avec les notes, d'abord en fin de 
volume, puis au bas de la page ${ }^{21}$. Cela conduit également à prendre en considération les formes d'inscriptions littérales ou graphiques, lorsque l'on a affaire à des langues où le rapport entre le contenu sémantique du mot et les formes graphiques n'est pas fixé ; cette prise en compte méticuleuse constitue un élément clé de l'interprétation.

Je me souviens d'un passage de Macbeth que commentent Peter Stallybrass et Margreta de Grazia où le mot heir/hair/air désigne, selon les éditions, l'héritier, les cheveux, ou l'air que l'on respire. Cette ambiguïté de la transcription écrite par rapport à quelque chose qui a été dit, et sans doute compris d'une manière ou d'une autre, renvoie à l'idée de la matérialité du texte. L'autre exemple, le plus fameux, étant «To be or not to be, aye there's the point ». Tel est le vers que l'on rencontre dans la première édition d'Hamlet de 1603 ; il n'est pas du tout question de that is the question. Cette variation, qui est si forte dans les œuvres théâtrales, et particulièrement dans le théâtre élisabéthain ou du premier $\mathrm{XVII}^{\mathrm{e}}$ siècle, donne une indication sur la nécessité d'étendre ce qui est pris en compte dans la description érudite, et de considérer que ce sont là des éléments fondamentaux pour la compréhension des conditions qui régissent la composition du texte, comme pour restituer ses interprétations variables et historiquement différenciées. Alors tout cela plaide, me semble-t-il, pour redonner à la bibliographie, devenue sociologie des textes, ou à la paléographie et à la codicologie, devenues histoire de la culture écrite ou comme le dirait Armando Petrucci histoire de la culture graphique, une centralité absolument évidente. On peut déplorer que l'histoire culturelle et la critique littéraire demeurent encore assez réticentes quant à ces apports.

En même temps, l'imprimé a tendance à renforcer l'autonomisation d'un domaine du littéraire et du savant dans le monde de l'écrit, par rapport à des écritures pratiques, administratives ou notariales, qui restent manuscrites. N'est-ce pas là une différence fondamentale avec le Moyen Âge, qui a des effets sur les chercheurs, puisque la proximité des études sur écrit pragmatique et écrit savant semble plus grande en histoire médiévale qu'en histoire moderne, où les recherches sur l'écrit portent avant tout sur l'écrit savant, voire spécifiquement sur le littéraire?

38 Je ne suis pas tout à fait d'accord avec la fin de votre question. Il me semble que dans l'histoire moderne, un des mouvements importants a été de transformer ce qui était considéré fondamentalement comme une source en objet même du travail, ce qui revient à dire que les recherches sur l'écrit portent aussi sur des écrits pragmatiques, documentaires et pratiques. Bien que demeurés minoritaires par rapport à l'utilisation documentaire des écrits notariés, je pense par exemple aux travaux sur les conditions mêmes de l'établissement des inventaires après décès. Le conflit, la présence des héritiers créent une dynamique que l'analyse du document peut restituer. On pourrait dire la même chose de la nouvelle manière d'aborder les archives judiciaires, non plus pour construire une statistique de la criminalité, mais comme le lieu d'un dialogue inégal entre le juge, l'accusé et les témoins, et comme le lieu de la transcription de la parole vive. Le document judiciaire à l'intérieur de la procédure du procès se retrouve interrogé pour lui-même, comme une pratique de l'écrit qui, par la réécriture et parfois par la traduction, s'empare et déforme la parole de l'accusé ou du témoin. Le débat suscité par Montaillou ${ }^{22}$ portait précisément sur la mise entre parenthèses, dans la démarche d'Emmanuel Le Roy Ladurie qui entendait aller droit au vécu, des conditions de production du document et de toutes les médiations à l'œuvre dans son écriture. On pourrait penser aussi aux archives de police telles que les traitent des historiens 
comme Arlette Farge qui, au-delà de leur utilisation documentaire et immédiate pour une histoire sociale des existences, posent la question des conditions mêmes de leur production intellectuelle et matérielle. Donc il me semble que certains travaux sur les écritures documentaires ont réintroduit la dimension de l'étude de leurs conditions de production.

Par ailleurs, je pense qu'il y a aussi dans le monde de l'histoire moderne et contemporaine un intérêt puissant pour ce qui a été appelé, peut-être d'une manière un peu maladroite, les écritures ordinaires. Le livre dirigé par Daniel Fabre ${ }^{23}$ a abordé ces questions depuis les écritures des carnets, des cahiers, des livres de comptes, des journaux - au sens de mémoires -, pour aller jusqu'à la correspondance. Il s'agit d'un énorme chantier de travail lié, pour l'époque moderne et contemporaine, à ces écritures sans qualités. Jean Hébrard avait proposé la constitution d'une sorte de bibliographie matérielle de ces objets. Il s'agit en effet souvent d'objets qui, sur un même support, enchaînent des genres différents (livres de comptes, journaux, mémoires...). L'histoire de l'écriture épistolaire évidemment serait un assez bon exemple, pour le Moyen Âge, d'un écrit plus savant, régi par des modèles ou des normes et dont les producteurs sont des lettrés. Pour la période moderne, le repérage des correspondances ordinaires, à distance de toute application de normes et de toute mobilisation d'une culture lettrée, est parfois difficile. Mais dans les dix ou les quinze dernières années, est apparu, je pense, un intérêt pour un écrit pragmatique, pratique et ordinaire qui nuance le contraste entre le Moyen Âge et l'époque moderne, même si, effectivement, comme on a pu l'évoquer, dans certains travaux sur le Moyen Âge, le continuum et la parenté morphologique entre les écritures documentaires et les écritures savantes ou «littéraires" ont pu être un objet d'études plus fortement analysé que dans le monde moderne.

Je pense que des travaux comme ceux de Philippe Artières, de Béatrice Fraenkel ou de Daniel Fabre ont défini un espace pour ce travail sur les écritures ordinaires, entendu non pas exclusivement comme livrant des informations. Par exemple, un livre écrit par trois de mes collègues de l'EHESS. qui s'appelait Ces bonnes lettres ${ }^{24}$ repère, dans l'iconographie et dans les lettres elles-mêmes, les représentations de leurs conditions de production et déplace l'attention des catégories d'affectivité ou des informations factuelles, vers la manière dont les lettres énoncent elles-mêmes les perspectives et les conditions qui ont régi leur écriture.

41 Un livre écrit par Lodovica Braida sur les manuels épistolaires dans la Renaissance italienne porte l'attention sur le fait que ce genre, tourné vers la proposition de modèles qui définissent des normes, était le lieu où a subsisté, le plus tardivement, l'hétérodoxie ${ }^{25}$. Dans ces recueils de lettres données comme modèles, sans doute parce qu'ils pouvaient être moins immédiatement suspectés que d'autre genres, a subsisté, jusque assez tard dans le $\mathrm{xVI}^{\mathrm{e}}$ siècle, tout ce monde des hétérodoxes - décrit Silvana Seidel Menchi ${ }^{26}$-, qui ne sont pas vraiment des luthériens, même si on le prétend, mais qui se situent à distance de l'orthodoxie catholique. On a là un exemple intéressant de relations entre un genre pragmatique et une affirmation intellectuelle ou spirituelle. Je crois que la question que vous posez peut porter à multiplier ce type d'analyses et donc du coup à rapprocher ce qui est, peut-être, encore trop séparé.

Une autre manière de faire consiste à comprendre comment dans des textes relevant de l'écriture savante sont appropriées des pratiques ou des objets qui appartiennent aux écritures ordinaires, ou à la cultura grafica telle que l'entend Armando Petrucci. Une 
autre stratégie peut consister à remonter du document à sa production, porter attention à ses sources plus difficiles à retrouver parfois, mais qui forment ce continent qu'on va appeler les écritures ordinaires ou pragmatiques, et à repérer, morphologiquement, des croisements entre des genres. Les œuvres de fiction peuvent ainsi s'approprier des contenus, des pratiques ou des objets de l'écriture ordinaire ; et les modèles épistolaires, des prises de positions idéologiques, spirituelles ou intellectuelles.

Cet isolement d'une sphère littéraire pose le problème de l'auteur, qui au Moyen Âge est à la fois une question transversale à la production de manuscrits et d'actes et inextricablement liée à celle de l'autorité : à l'époque moderne, la notion d'auteur semble se particulariser, ce qui rejoint les observations de Michel Foucault sur ce point ${ }^{27}$. Pensez-vous que l'histoire de la fonction auteur soit une manière possible de périodiser le rapport à l'écrit d'une société ? En miroir de cette fonction auteur, pensez vous que l'on puisse également écrire l'histoire et la périodiser à travers la figure du lecteur qui en est en quelque sorte le pendant et qui se multiplie, à partir de la fin du Moyen Âge, avec l'apparition d'un lectorat non professionnel?

La question pose d'abord en amont, on l'a évoqué souvent, la difficulté - encore plus forte pour vous médiévistes - d'utiliser cette catégorie du " littéraire " pour désigner tout un répertoire de textes que l'on suppose détachés de la fonction religieuse et spirituelle, politique et savante. Évidemment, c'est le premier problème sur lequel on bute. On le voit bien dans l'essai de Jacqueline Cerquiglini qui ouvre une histoire de la littérature française publiée récemment sous la direction de Jean-Yves Tadiée ${ }^{28}$. Elle se confronte avec cette définition de la littérature, non pas que le mot n'existe pas mais il désigne, même au-delà de l'époque médiévale, des corpus de textes que nous ne désignerions pas aujourd'hui par le terme de "littéraires ». D'autre part, des corpus que nous désignons comme participant de l'histoire de la littérature n'avaient aucunement ce statut ou cette désignation antérieurement. Si vous prenez les dictionnaires de la langue aux xVII ${ }^{\mathrm{e}}$-xVIII ${ }^{\mathrm{e}}$ siècles, le Richelet, le Furetière, l'Académie, le mot « littéraire » désigne l'érudition et « littérature » désigne parfois même les savoirs scientifiques. Il y a donc une très grande confusion autour de la notion de littérature qui a été réduite par le moment hérité du xviII ${ }^{e}$ siècle durant lequel un certain nombre de critères ont été stabilisés, et dont nous sommes les héritiers. D'abord le primat du critère de l'originalité dans la production d'une fable ou d'une fiction. Le fait, comme vous l'avez dit, que cette originalité soit rapportée à de l'individualité et donc à un auteur qui projette, dans cette originalité, sa manière unique de penser, de sentir ou d'écrire. Le troisième élément est la propriété de l'auteur sur son texte, même lorsqu'il le cède au libraire-éditeur. Originalité, individualité et propriété définissent le régime $\mathrm{du}$ fonctionnement de la littérature et rendent l'usage de ce concept, qui charrie implicitement ces trois éléments, très difficile pour des périodes où aucun de ces trois éléments n'est présent.

En effet - et je ne m'aventurerai pas dans le Moyen Âge - à l'époque des $\mathrm{XVI}^{\mathrm{e}}$ et XVII siècles, pour de nombreux genres, le critère de l'originalité est tout à fait second à côté du critère de l'imitatio, qui n'est ni reproduction à l'identique, ni assimilable au surgissement romantique de l'originalité, mais constitue une pratique où s'insinue une différence. Deuxièmement, l'individualité est seconde par rapport à l'écriture à plusieurs mains. Troisièmement, l'auteur ne peut revendiquer aucune propriété sur son texte, ni économique, puisque par exemple dans le cas du théâtre il appartient aux compagnies, ni intellectuelle puisque, si le délit de contrefaçon est constitué 
juridiquement à l'intérieur des législations royales ou des pratiques des communautés des libraires et des imprimeurs, il n'en est absolument pas de même pour celui de plagiat. Cela ne veut pas dire que l'on ne peut pas protester d'une certaine manière quand on pense que son invention littéraire, à l'intérieur de l'imitatio, a été reproduite par un autre. Mais cette protestation ne peut pas se faire sur la base d'un délit juridiquement constitué. On a là un univers qui ne se laisse pas apprivoiser par la catégorie du littéraire. En témoignent les frontières très mobiles de ce que l'on inclut lorsque l'on écrit des histoires de la littérature. Dans ce livre récent, dont nous avons déjà parlé29, Frank Lestringant inclut, pour le $\mathrm{xvI}^{\mathrm{e}}$ siècle, les récits de voyage, la rhétorique politique ou spirituelle, qui exceptionnellement faisaient partie du corpus de la littérature. Il y a donc une variabilité, qui constitue l'une des difficultés les plus grandes, parce que si l'on ne perçoit pas cette variabilité, on introduit une invariance de la littérature, une définition rétrospective du corpus, et une représentation anachronique des conditions de production des fictions. La notion d'auteur peut être utile à condition de la débarrasser des critères de spécificité, d'originalité et de propriété qui lui sont attribués à partir du XVIII ${ }^{\mathrm{e}}$ siècle. Ce que Michel Foucault disait peut sans doute impliquer cela, mais on pourrait aussi le résumer d'une manière plus élémentaire: dans une société donnée, quels sont les discours qui dans leur fonctionnement supposent la référence au nom propre ? L'auteur est finalement ce qui permettait de discriminer, dans une même société, des discours qui ne supposent aucunement cette fonction auteur et d'autres qui l'exigent. Et, d'autre part, l'analyse de Michel Foucault permettait d'ébaucher une hypothèse chronologique de longue durée. $\mathrm{Au}$ début de l'époque moderne, s'opérait pour lui un basculement - sans doute très discutable -, où les textes de fiction, qui supposaient l'auteur et devaient être rapportés au nom propre, se distinguaient des discours de savoir dont la validité procédait en quelque sorte de leur présence à l'intérieur d'un système d'énoncés accrédités d'emblée comme scientifiques.

Évidemment la discussion peut s'ouvrir, parce que d'une part, comme j'essayais de dire, il y a de nombreux genres et de nombreuses formes de textes de fictions qui, aux $\mathrm{xvI}^{\mathrm{e}}$ $\mathrm{XVII}^{\mathrm{e}}$ siècles, ne sont pas immédiatement liés à cette assignation au nom propre de leur auteur et, à l'inverse, l'époque médiévale et l'époque moderne se caractérisent par une diffraction de la fonction auteur comme garante de l'autorité d'un texte de savoir. Cette fonction peut être attribuée, comme l'a montré Mario Biagioli ${ }^{30}$, au dédicataire du texte - patron ou souverain -, ou au libraire éditeur, ou au traducteur. Le nom propre peut également être uniquement un marqueur, lorsque les noms propres sont associés - comme Michel Foucault l'évoque - à des lois de la physique ou de la chimie au XIX ${ }^{e}$ siècle.

Il convient aussi de considérer les procédures par lesquelles des textes deviennent des œuvres, à partir du moment où ils sont assignés à un nom propre d'auteur. $\mathrm{Au}$ XVIII ${ }^{\mathrm{e}}$ siècle, cette assignation se fait fondamentalement à partir d'un critère juridique, celui de la propriété littéraire reconnue comme telle et fondant les revendications du copyright. L'assignation du texte au nom propre a été dépendante des procédures juridiques liées à la propriété, mais aussi, préalablement, des procédures judiciaires liées à l'autorité. Ce lien apparait dans les textes des censures des Index de l'Inquisition. Et puis, si l'on revient à notre libro unitario, existait une assignation qui ne provenait ni du droit de propriété, ni des pratiques de la censure et de la prohibition, mais résultait d'une perception culturelle qui donnait unité à des textes à l'intérieur d'un même livre. 
On retrouve ici la question de la reliure, au sens matériel et métaphorique. Je crois que la question de la fonction auteur permet de montrer cette discontinuité, mais à condition à la fois de reprendre la distinction matricielle de Michel Foucault - tout texte a été écrit par quelqu'un mais tous les textes n'ont pas un auteur -, tout en se gardant d'être prisonnier de son esquisse chronologique parce que, finalement, ce n'est pas ce qui l'intéressait le plus. À gros traits, il a dessiné l'inversion d'assignation de l'auteur et il a porté son attention sur un moment clé, le XVIII siècle, avec l'entrée des œuvres dans le régime de la propriété des choses. Et on a pu voir rétrospectivement, comme l'a montré une nouvelle histoire des sciences, ainsi que la réflexion juridique sur les catégories de la propriété des objets écrits, que l'on est devant une réalité plus complexe. Une autre voie d'entrée est évidemment de reprendre toutes les taxinomies qui classent, hiérarchisent des genres de discours dans les différents moments du temps, sans que n'y apparaisse nécessairement le critère de littérature, ou lorsqu'il apparait, avec des significations inversées de la nôtre.

Il s'agit évidemment d'une question délicate qui suscite une résistance assez forte de la critique littéraire. Je n'ai pas de solution mais je pense que c'est un objet ouvert et très foucaldien dans un sens. D'un côté on est dépendant de mots qui deviennent des concepts lorsqu'ils sont figés en un certain nombre de traits distinctifs - et nous sommes amenés à les manier - et, en même temps, ces concepts produisent des effets d'invariance rétrospective, là où le projet foucaldien, ou tout projet historien quel qu'il soit, reconnaît des discontinuités ou des successions de configuration. Comment négocier ce rapport entre l'inertie des mots, qui conduit à l'invariance des notions, et d'un autre côté la profonde discontinuité des objets, des pratiques, des représentations et des catégories ? Je crois qu'il s'agit d'un défi tout à fait redoutable, particulièrement fort dans le cas de l'usage du terme littérature.

Puisque nous voilà engagés dans des considérations historiographiques liées aux pratiques de l'écrit, vous faites référence dans votre leçon inaugurale à Lucien Febvre "qui se moquait de ces historiens dont "les paysans, en fait de terre grasse, semblaient ne labourer que de vieux cartulaires" ». On voit bien ce qu'il vise, et en même temps, ne pensez-vous pas que c'est ce genre de position qui a fait passer à l'arrière-plan la réflexion sur la construction matérielle et textuelle des documents?

Évidemment, ce que disait Lucien Febvre présente des risques, vous avez raison. Ce qu'il voulait dire c'est qu'il existait des sources pour saisir les pratiques qui débordaient la documentation écrite et il proposait de la sorte une ouverture vers une histoire des paysages, vers une archéologie médiévale ou moderne des objets. Il voulait, dans le langage imagé du temps, qui nous paraît peut-être un peu désuet aujourd'hui, « ouvrir les fenêtres ». Dans une conférence faite aux élèves de l'École normale supérieure, il proposait de sortir des bibliothèques, des documents écrits et de cette érudition qui avait, à l'inverse de votre crainte, marqué comme une sorte d'écran entre la réalité passée des choses et sa compréhension. J'avais repris cette image comme une sorte de paradoxe dans la leçon puisque, dans un sens, j'appelais à labourer dans les cartulaires, à s'enfouir dans les objets écrits, avec cependant pour dessein la revendication d'une histoire des pratiques et des interprétations. Il s'agissait d'une objection adressée à moi-même. Avec cette idée de définir un programme de recherches à partir des écrits, des traces et des objets qui les portent, le risque pourrait apparaître d'enfermer dans cet écrit les pratiques qui s'en sont saisies; et de substituer de la sorte à l'histoire des pratiques, une histoire des textes, même augmentée d'une attention tournée vers les 
objets qui les portent. Je pense qu'il y a là une mise en garde salutaire; et vous avez raison de dire qu'elle ne doit pas se transformer en une tentation de négliger les dispositifs que l'on rencontre dans les documents écrits et qui sont à la fois révélateurs de leurs conditions sociales de production et des conflits, des transactions et des négociations qui les produisent. On l'a évoqué pour les archives de police, les archives judiciaires, mais une édition imprimée est aussi le résultat de ces transactions, négociations, incompréhensions, compétitions qui viennent s'inscrire dans les dispositifs les plus matériels de l'objet. Il faut donc lire le texte-objet avec cette préoccupation. Voilà une première réponse à la crainte exprimée.

51 Un deuxième élément de réponse est la mise en dialogue de ces objets avec d'autres sources ; il est possible de bâtir, à partir d'eux, une histoire des pratiques, au moins à travers les représentations qui en sont données par tous les discours qui entendent les normer, les prescrire, les proscrire, les décrire, les critiquer... Donc l'analyse, dans ce rapport aux pratiques, doit considérer les textes à travers une procédure descriptive et analytique qui rend compte de leur production. Elle-même doit d'emblée être installée dans le terreau de la conflictualité sociale. D'autre part, ces cartulaires ou ces éditions et les textes qu'elles portent sont en même temps des sources qui ont un statut de représentation. Et l'écriture historique est elle-même une représentation du passé qui utilise ces représentations préalables. On peut se référer ici aux analyses de Paul Ricœur dans La mémoire, l'histoire, l'oubli $i^{31}$. Du coup, ce qui devient capital dans la question, c'est de penser que par le déchiffrement rigoureux des procédures qui construisent dans les documents les représentations des réalités, des pratiques ou des pensées - qu'ils soient au plus près du pragmatique ou plus élaborés esthétiquement ou intellectuellement -, on a une chance de produire une lecture qui ne déduise pas le réel disparu des représentations qui en sont données, sans mettre radicalement en cause l'idée d'une possible connaissance de ce passé. S'ouvre de la sorte une voie entre deux écueils : le premier désigné, je crois, par Lucien Febvre, le second présent dans les débats autour du post-modernisme et surtout d'un retour du relativisme et du scepticisme dans la problématique historienne. Pour parodier les formules de Louis Marin, on pourrait dire que c'est seulement l'analyse des pratiques de la représentation (incluant le savoir érudit sur les objets) qui permet de s'approcher sans scepticisme, ni naïveté, des représentations des pratiques. Cette articulation est bien au cœur de la question que l'on pose et qui déborde finalement la polémique de Lucien Febvre vis-àvis de l'École des chartes, comme l'enfermement dans une textualité dont on ne peut pas sortir - «il n'y a pas de hors texte ». Simplement je crois que lorsque l'on pense à l'analyse des pratiques de la représentation, il faut élargir le questionnaire avec les éléments que l'on a évoqués aujourd'hui. Cette analyse, qui commence avec les données les plus techniques, est la seule qui permette un déchiffrement contrôlé des pratiques représentées.

\section{NOTES}

1.R. CHARTIER, Écouter les morts avec les yeux, Paris, 2008. 
2..Voir par exemple N. CANNATA, Il canzoniere a stampa (1470-1530). Tradizione e fortuna di un genere fra storia del libro e letteratura, Rome, 2000.

3..F. ALESSIO, « Conservazione e modelli di sapere nel Medioevo », La memoria del sapere. Forme di conservazione e strutture organizzative dall'Antichità a oggi, P. ROSSI éd., Rome-Bari, 1988, p. 99-133.

4.L. FEBVRE et H.-J. MARTIN, L'apparition du livre, Paris, 1958.

5..E. EISENSTEIN, The Printing Press as an Agent of Change. Communications and Cultural Transformations in Early Modern Europe, Cambridge-Londres-New-York, 1979.

6.E. EISENSTEIN, The Printing Revolution in Early Modern Europe, Cambridge-New York, 1983 [trad. fr. : La révolution de l'imprimé à l'aube de l'époque moderne, Paris, 1991].

7..A. PETRUCCI, « Minuta, autografo, libro d'autore », Il libro e il testo, C. QUESTA et R. RAFFAELI éd., Urbino, 1984, p. 339-414.

8.A. PETRUCCI, La Scrittura : ideologia e rappresentazione, Turin, 1986 [trad. fr. : Jeux de lettres : formes et usages de l'inscription en Italie (XI'-XXe siècles), Paris, 1993].

9.J. GOODY, La raison graphique : la domestication de la pensée sauvage, Paris, 1978 ; ID., Entre l'oralité et l'écriture, Paris, 1994 ; ID., Pouvoirs et savoirs de l'écrit, Paris, 2007.

10.M. CLANCHY, From Memory to Written Record, England 1066-1307, Oxford-Cambridge, 1993 ².

11.R. CHARTIER, «Culture écrite et littérature à l'âge moderne », Annales HSS, vol. 4, 2001, p. 783-802.

12.ID., « Culture, Lumières, doléances : les cahiers de 1789 », Revue d'Histoire moderne et contemporaine, t. 28, 1981, p. 68-93.

13.BAUDRI de BOURGUEIL, Poèmes, éd. trad. et com. J.-Y. TILLIETTE, 2 vol., Paris, 1998-2002.

14.R. CHARTIER, Inscrire et effacer. Culture écrite et littérature, XIe-XVIII' siècle, Paris, 2005.

15.ID., Culture écrite et société. L'ordre des livres (XIV'-XVIII' siècle), Paris, 1996.

16.E. R. CURTIUS, Europäische Literatur und lateinisches Mittelalter, Berne, 1948 [trad. fr. La littérature européenne et le Moyen Âge latin, Paris, 1956, ici, p. 368-428].

17.J. CERQUIGLINI-TOULET, La couleur de la mélancolie : la fréquentation des livres au XIVe siècle (1300-1415), Paris, 1993.

18..P. STALLYBRASS, R. CHARTIER et J. FRANKLIN MOWERY, « Hamlet's Tables and the Technologies of Writing in Renaissance England», Shakespeare Quarterly, t. 55/4, 2004, p. 379-419.

19.S. FREUD, « Notes sur le bloc-notes magique », Résultats, idées, problèmes 2 (1921-1938), Paris, 1985, p. 119-124.

20.D. F. MCKENZIE, Bibliography and the Sociology of Texts, Londres, 1986 [trad. fr. : La bibliographie et la sociologie des textes, Paris, 1991].

21..A. GRAFTON, The Footnote : a Curious History, Londres, 1997 [trad. fr. Les origines tragiques de l'érudition : une histoire de la note en bas de page, Paris, 1998].

22.E. LE ROY LADURIE, Montaillou, village occitan de 1294 à 1324, Paris, 1975.

23. Par écrit : ethnologie des écritures quotidiennes, D. FABRE dir., Paris, 1997.

24. Ces bonnes lettres : une correspondance familiale au XIXe siècle, C. DAUPHIN, P. LEBRUN-PÉZERAT et D. POUBLAN éd., Paris, 1995.

25.L. BRAIDA, Le raccolte epistolari del Cinquecento tra inquietudini religiose e "buon volgare », Rome, 2009.

26.S. SEIDEL MENCHI, Erasmo in Italia (1520-1580), Turin, 1987 [trad. fr. : Érasme hérétique : Réforme et Inquisition dans l'Italie du XVI siècle, Paris, 1996].

27.M. FOUCAULT, «Qu'est-ce qu'un auteur? », Bulletin de la société française de philosophie, 3, 1969, p. 73-104 [repris dans Dits et écrits 1954-1988. I. 1954-1975, Paris, 2001]

28.J. CERQUIGLINI-TOULET, « La littérature médiévale », La littérature française : dynamique \& histoire, J.Y. TADIÉ dir., Paris, 2007, vol. 1, p. 27-204. 
29. Voir le volume cité à la note précédente.

30.M. BIAGIOLI, Galileo, Courtier. The Practice of Science in the Culture of Absolutism, Chicago, 1993.

31.P. RICEUR, La mémoire, l'histoire, l'oubli, Paris, 2000. 